\title{
ON COEFFICIENTS OF CIRCUIT POLYNOMIALS AND CHARACTERISTIC POLYNOMIALS
}

\author{
E. J. FARRELL \\ Department of Mathematics \\ The University of the West Indies \\ St. Augustine, Trinidad \\ (Received May 10, 1984)
}

IBSTRACT. Results are given from which expressions for the coefficients of the simple circuit polynomial of a graph can be obtained in terms of subgraphs of the graph. From these are deduced parallel results for the coefficients of the characteristic polynomial of a graph. Some specific results are presented on the parities of the coefficients of characteristic polynomials. A characterization is then determined for graphs in which the number of sets of independent edges is always even. This leads to an interesting link between matching polynomials and characteristic polynomials.

Finally explicit formulae are derived for the number of ways of covering two well known families of graphs with node disjoint circuits, and for the first few coefficients of their characteristic polynomials.

KEY WORDS AND PHRASES. Circuit polynomials, circuit cover of a graph, simple circuit polynomial, hamiltonian circuit, characteristic polynomial.

1380 MATHEMATICS SURJECT CLASSIFICATION CODES. O5A15, $05 C 99$.

\section{INTRODUCTION}

The graphs considered here will be finite and will have no loops or multiple edges. Let $G$ be such a graph. By a circuit cover (or simply a cover) in $G$ will mean a spanning subgraph of $G$ whose components are all circuits. We will take the circuit with one node to be an isolated node, and the circuit with two nodes to be an edge of $G$. With each circuit $\alpha$ in $G$ associate an indeterminate or weight $w_{\alpha}$, and with each cover $C$ the weight

$$
w(C)=\prod_{\alpha} w_{\alpha}
$$

where the product is taken over all the components of $C$. Then the circuit polynomial of $G$ is $\sum w(C)$, where the summation is taken over all the circuit covers in $G$.

If we give each circuit with $r$ nodes a weight $w_{r}$, then the circuit polynomial of $G$ is written as $C(G ; \underline{w})$, where $\underline{w}=\left(w_{1}, w_{2}, w_{3}, \ldots\right)$. If we now put $w_{i}=w$ for all $i$, then the resulting polynomial, denoted by $\mathrm{C}(\mathrm{G} ; \mathrm{w})$, is called the simple circuit polynomial of $G$. In this case,

$$
C(G ; w)=\sum_{k=0}^{p} a_{k} w^{p-k} \text {, }
$$


where $p$ is the number of nodes in $G$, and $a_{k}$ is the number of covers of $G$ with $p-k$ components. The basic properties of circuit polynomials have been discussed in Farrel1 [2].

Throughout this article, we will assume that the graph $G$ contains $p$ nodes and $q$ edges, unless otherwise specified. First we will give a result which describes the possible circuit covers of $G$. We will then use this result to deduce explicit formulae for the coefficients of $\mathrm{C}(\mathrm{G} ; \mathrm{w})$ in terms of the circuit subgraphs of $G$. By applying a theorem which relates the circuit polynomial of a graph to its characteristic polynomial, we will deduce results about the parities of the coefficients of the characteristic polynomial of a graph. A new characterization will then be given for graphs in which the number of sets of independent edges is always even. We will then establish a connection between characteristic polynomials and matching polynomials (see Farre11 [3]). Finally, we apply our results to some well known graphs.

\section{PRELIMINARY RESULTS}

THEOREM 1. Let $G$ be a graph with p nodes. Then the circuit covers of $G$ with cardinality $\mathrm{p}-\mathrm{k}(\mathrm{k}>0)$ consist of $\mathrm{p}-\mathrm{c}$ isolated nodes, together with an $r_{1}-$ gon, an $r_{2}$-gon, ..., an $r_{s}$-gon, where $k+1 \leqq c \leqq 2 k, s=c-k$ and $\left(r_{1}, r_{2}, \ldots, r_{s}\right)$ is a partition of $c$ with each part greater than 1 .

PROOF. Let $C$ be a cover of $G$ with cardinality $p-k(k>0)$, and $x$ the number of isolated nodes in $C$. Let the non-trivial components of $c$ be $r_{i}$-gons, where $\mathbf{i}=1,2, \ldots, \mathrm{s}$. Then

$$
\sum_{i=1}^{s} r_{i}+x=p .
$$

Also $x+s=p-k$ which implies $x=p-(k+s)$. Put $k+s=c$. Also note that $0<s \leqq k$. The result follows.

Q.E.D.

Notice that if $G$ is a tree, then $r_{i}=2, \forall_{i}$ which implies that $G$ consists of $p-c$ isolated nodes and $\frac{c}{2}$ edges from which $i$ follows that $s=\frac{c}{2}=c-k$ so $c=2 k$. Hence a cover with cardinality $p-k$ will consist of $p-2 k$ isolated nodes and $k$ independent edges. It follows that $a_{k}$ will be the number of sets of $k$ independent edges in $G$. This leads to the following corollary.

COROLLARY 1.1. Let $\mathrm{T}$ be a tree with $\mathrm{p}$ nodes. Then

$$
C(T ; w)=\sum_{k=0}^{p-1} e_{k} w^{p-k},
$$

where $e_{k}$ is the number of sets of $k$ independent edges in $T$.

It is clear that Corollary 1.1 provides a lower bound for the coefficients of $C(G ; w)$. Thus we have

THEOREM 2. Let $G$ be a graph with $p$ nodes and $T$ a spanning tree of $G$. Let $a_{k}$ and $e_{k}$ be the coefficients of $w^{p-k}$ in $C(G ; w)$ and $C(T ; w)$, respectively. Then $a_{k} \geqq e_{k}$. 3. THE COEFFICIENTS OF CIRCUIT POLYNOMIALS.

We will use the notation $\mathrm{N}_{G}\left(n_{1}, n_{2}, \ldots, n_{k}\right)$ for the number of subgraphs of $G$ whose components are an $n_{1}$-gon, an $n_{2}-$ gon, $\ldots$, and an $n_{k}$-gon. The following result is clear from Theorem 1 . 
COROLLARY 1.2. Let $a_{k}$ be the coefficient of $w^{p-k}$ in $C(G ; w)$. Then

$$
a_{k}=\sum_{j=k+1}^{2 k} N_{G}\left(\pi_{j}\right),
$$

where $\pi_{j}$ is a partition of $j$ with $j-k$ parts and with each part greater than 1 .

Some specific values of $a_{k}$ are the following:

$$
\begin{aligned}
a_{0}= & 1 . \\
a_{1}= & N_{G}(2) \text { (the number of edges in } G . \\
a_{2}= & N_{G}(2,2)+N_{G}(3) . \\
a_{3}= & N_{G}(2,2,2)+N_{G}(2,3)+N_{G}(4) . \\
a_{4}= & N_{G}(2,2,2,2)+N_{G}(2,2,3)+N_{G}(2,4)+N_{G}(3,3)+N_{G}(5) . \\
a_{5}= & N_{G}(2,2,2,2,2)+N_{G}(2,2,2,3)+N_{G}(2,2,4)+N_{G}(2,3,3)+N_{G}(2,3) \\
& +N_{G}(3,4)+N_{G}(6) . \\
a_{6}= & N_{G}(2,2,2,2,2,2)+N_{G}(2,2,2,2,3)+N_{G}(2,2,2,4)+N_{G}(2,2,3,3) \\
& +N_{G}(2,2,5)+N_{G}(2,3,4)+N_{G}(2,6)+N_{G}(3,6)+N_{G}(3,3,3) \\
& +N_{G}(4,4)+N_{G}(7) .
\end{aligned}
$$

4. THE COEFFICIENTS OF CHARACTERISTIC POLYNOMIALS.

We will denote the characteristic polynomial of a graph $G$ by $\phi(G ; x)$.

The following theorem was proven in [2].

THEOREM 3. Let $G$ be a graph. Then $\phi(G ; x)$ can be obtained from $C(G ; w)$ by putting $\mathrm{w}_{1}=\mathrm{x}, \mathrm{w}_{2}=-1$ and $\mathrm{w}_{\mathrm{k}}=-2$, for $\mathrm{k}>2$.

If we assign weights to the covers suggested by Theorem 1, according to the number of nodes in their components, and then use the general expressions for $a_{k}$, we can obtain results which give the coefficients of the characteristic polynomial of a graph in terms of subgraphs of the graph. Such results could provide a useful check when finding characteristic polynomials of large graphs. They could also be used to find explicitly the characteristic polynomials of small graphs. For example, if

$$
\phi(G ; x)=\sum_{k=0}^{p} b_{k} x^{p-k},
$$

then

$$
\begin{aligned}
b_{0}= & 1, \quad b_{1}=0, \\
b_{2}= & -N_{G}(2), \\
b_{3}= & -2 N_{G}(3), \\
b_{4}= & N_{G}(2,2)-2 N_{G}(4), \\
b_{5}= & 2 N_{G}(2,3)-2 N_{G}(5), \\
b_{6}= & -N_{G}(2,2,2)+2 N_{G}(2,4)+4 N_{G}(3,3)-2 N_{G}(6), \\
b_{7}= & -2 N_{G}(2,2,3)+2 N_{G}(2,5)+4 N_{G}(3,4)-2 N_{G}(7), \\
b_{8}= & N_{G}(2,2,2,2)-2 N_{G}(2,2,4)-4 N_{G}(2,3,3)+2 N_{G}(2,6) \\
& +4 N_{G}(3,5)+4 N_{G}(4,4)-2 N_{G}(8),
\end{aligned}
$$




$$
\begin{aligned}
& \mathrm{b}_{9}=-2 \mathrm{~N}_{G}(2,2,2,3)-2 \mathrm{~N}_{G}(2,2,5)-4 \mathrm{~N}_{G}(2,3,4)-8 \mathrm{~N}_{G}(3,3,3) \\
& \quad+2 \mathrm{~N}_{G}(2,7)+4 \mathrm{~N}_{G}(3,6)+2 \mathrm{~N}_{G}(4,5)-2 \mathrm{~N}_{\mathrm{G}}(9), \\
& \text { etc. }
\end{aligned}
$$

The next corollary arises from Theorem 3 and Corollary 1.1 (with general weights $\mathrm{w}_{1}$ and $\mathrm{w}_{2}$ ).

COROLLARY 3.1. Let $T$ be a tree with $p$ nodes. In $\phi(T ; x)$ the coefficient of $\mathrm{x}^{\mathrm{p}-2 \mathrm{k}}$ is negative if $\mathrm{k}$ is odd and positive if $\mathrm{k}$ is even.

The following theorems give information on the parities of the coefficients of the characteristic polynomials of graphs. These results can provide a useful check when calculating characteristic polynomials. As far as we know these results are new.

THEOREM 4. Let $G$ be a graph with $p$ nodes. The coefficient of $x^{p-2 k-1}(k \geqq 0)$ in $\phi(G ; x)$ is even.

PROOF. It is clear from Theorem 3 that a term in $x^{p-2 k-1}$ could arise if and only if the associated monomial in $C(G ; w)$ contains the factor $w_{1}{ }^{p-2 k-1}$. Let $r$ be the index of $w_{2}$ in the monomial. Then, in the corresponding cover in $G, p-2 k-1$ nodes are isolated and $2 r$ of the remaining $2 k+1$ nodes are "used up" in forming independent edges. Since $2 r \neq 2 k+1$ for any values of $k$ and $r$, the monomial must contain terms $w_{i}^{s}$, where $s>0$ and $i>2$. Thus the coefficient of $x^{p-2 k-1}$ in $\phi(G: x)$ must contain a factor 2. The result now follows.

THEOREM 5. The coefficient of $x^{p-2 k}(k>0)$ is odd if and only if $G$ has an odd number of sets of $k$ independent edges.

PROOF. A term in $x^{p-2 k}$ in $\phi(G ; x)$ arises from terms in $C(G ; w)$ which contain a factor of $w_{1}{ }^{p-2 k}$. The remaining monomial of such a term may or may not contain a factor $w_{i}$, for $i>2$. We will consider two cases: (i) None of the associated terms in $C(G ; \underline{w})$ contain a factor $w_{i}$, for $i>2$; and (ii) there is an associated term containing a factor $w_{i}$, for $i>2$.

CASE (i). In this case, the associated term will be $e_{k} w_{1}{ }^{p-2 k} w_{2}{ }^{k}$, where $e_{k}$ is the number of sets of $k$ independent edges in $G$. The corresponding term in $\phi(G ; x)$ will be $(-1)^{k} e_{k} x^{p-2 k}$. This coefficient is odd if and only if $e_{k}$ is odd.

CASE (ii). Any associated term of $C(G ; w)$ which contains a factor $w_{i}$, for $i>2$, will give rise to an even coefficient of $x^{p-2 k}$ in $\phi(G ; x)$ (from Theorem 3 ). Hence the sum $s_{k}$ of the contributions of these terms will be even. If a term does not contain such a factor then it will be of the form discussed in Case (i). The resulting coefficient of $x^{p-2 k}$ will therefore be $(-1){ }^{k} e_{k}+s_{k}$; which is odd if and only if $e_{k}$ is odd. This completes the proof.

Q.E.D.

The following theorem combines the results of Theorems 4 and 5 . It gives an interesting characterization of graphs which have only even numbers of sets of independent edges.

THEOREM 6. Let $G$ be a graph with $p$ nodes. Then $G$ has an even number (possibly zero) of sets of $k$ independent edges for all values of $k>0$ if and only if all the coefficients in $\phi(G ; x)$ (except, of course, that of $x^{p}$ which is always 1 ) are even.

PROOF. Let us assume that $G$ has zero or an even number of sets of $k$ independent edges for all values of $k>0$. From Theorem 5 the coefficient of $x^{p-2 k}$ in $\phi(G ; x)$ will 
be zero or even for all values of $k>0$. By Theorem 4 the coefficient of $x^{p-2 k-1}$ will be zero or even, for all values of $k \geqq 0$. Hence all the terms in $\phi(G ; x)$ (except $x^{p}$ ) will have even coefficients.

Conversely, let us assume that all the coefficients (except that of $x^{p}$ ) in $\phi(G ; x)$ are even. Then it follows from theorem 5 that $G$ must have zero or an even number of sets of $k$ independent edges for all values of $k$. This completes the proof.

Suppose that $\underline{\text { Q.E.D. }}$ term $\mathrm{w}_{2}{ }^{\mathrm{r}}$ in the associated circuit polynomial cannot "use up" the $2 \mathrm{k}+1$ nodes (see proof of Theorem 4). It follows that the only terms with non-zero coefficients in $\phi(G ; x)$ are those in $x^{p-2 k}$. Since $p-2 k$ is odd if and only if $p$ is odd, we deduce the following result for trees.

THEOREM 7. Let $T$ be a tree with an odd (even) number of nodes. Then $\phi(T ; x)$ contains only odd (even) powers of $x$.

This result is also given in Harary et al [4].

The following corollary of Theorem 6 establishes an interesting connection between matching polynomials and characteristic polynomials of graphs.

COROLLARY 6.1. The matching polynomial of a graph $G$ contains only even coefficients (excluding that of $\mathrm{w}_{1}{ }^{\mathrm{p}}$ ) if and only if the characteristic polynomial of $\mathrm{G}$ contains only even coefficients (excluding that of $x^{p}$ ).

PROOF. Let us denote the matching polynomial of $G$ by $M(G ; w)$. Then the coefficient of ${ }{ }_{1}{ }^{p-2 k}{ }_{w_{2}}{ }^{k}(k \neq 0)$ is the number of sets of $k$ independent edges in $G$. This is even if and only if $G$ has an even number of sets of $k$ independent edges. Hence all the coefficients of. $M\left(G ; \underline{w}\right.$ ) (except that of $w_{1}{ }^{p}$ ) are even if and only if $G$ has an even number of sets of $k$ independent edges for all values of $k$. It follows that all the coefficients in $\phi(G: x)$ (except that of $x^{p}$ ) are even (by Theorem 6).

One previously known link between the two types of polynomials is that the matching polynomial and characteristic polynomial of a tree coincide, except for differences in the signs of the coefficients (see [2], Theorems 6 and 8).

Some of our results about characteristic polynomials can be derived from results given by Sachs [5]. However the circuit polynomial approach used here is basically simpler. It also demonstrates the usefulness of circuit polynomials as a device for investigations into properties of characteristic polynomials.

5. APPLICATIONS.

\section{The Whee1}

We define the wheel $W_{p}$ to be the graph obtained by joining an isolated node to all the nodes of a circuit with $\mathrm{p}-1$ nodes. We will call the isolated node the hub of $\mathrm{w}_{\mathrm{p}}$; the circuit, the rim of $\mathrm{W}_{\mathrm{p}}$; and an edge joining the hub to the rim a spoke. The following lemmas will be useful.

LEMMA 1 .

$$
N_{W_{P}}(n)=\left\{\begin{array}{cl}
p-1 & \text { if } n<p-1, \\
1 & \text { if } n=p-1, \\
0 & \text { if } n>p-1 .
\end{array} \text { for } n>2 .\right.
$$




$$
\mathrm{N}_{\mathrm{W}_{\mathrm{P}}}(2,2,2, \ldots, 2, \mathrm{n})=\left(\begin{array}{c}
\mathrm{p}-\mathrm{n}-\mathrm{k} \\
\mathrm{k}
\end{array}\right)(\mathrm{p}-1),
$$

where $k>0$ is the number of independent edges and $n>2$.

$$
\begin{aligned}
& N_{W_{P}}\left(n_{1}, n_{2}, \ldots, n_{k}\right)=0 \text { if } n_{i}>2 \text { and } n_{j}>2 \text { for some } i \text { and } j \text {, where } \\
& 1 \leqq i, j \leqq k .
\end{aligned}
$$

PROOF.

(i) The n-gon must contain two spokes and can "begin" with any of the p-1 spokes if $n<p-1$. If $n=p-1$, then the only $n$-gon will be the rim.

(ii) Since the $n$-gon must contain the hub, the $k$ independent edges must be chosen from the remaining subgraph, which will be a path with $p-n$ nodes. The number of ways of choosing these edges in $\left(\begin{array}{c}p-n-k \\ k\end{array}\right)$, from Theorem 7 of [2]. Since there are $p-1$ n-gons, the result follows.

(iii) Since there is only one hub and each $n$-gon $(n>2)$ must contain the hub, the result follows.

LEMMA 2.

$$
\mathrm{N}_{\mathrm{W}_{\mathrm{P}}}(2,2,2, \ldots, 2)=(\mathrm{p}-1)\left[\left(\begin{array}{c}
\mathrm{p}-\mathrm{k}-1 \\
\mathrm{k}-1
\end{array}\right)+\frac{1}{\mathrm{k}}\left(\begin{array}{c}
\mathrm{p}-\mathrm{k}-2 \\
\mathrm{k}-1
\end{array}\right)\right],
$$

where $\mathrm{k}$ is the number of independent edges.

PROOF. The sets of independent edges can be put into two classes: (i) those contain a spoke and (ii) those which do not contain a spoke. If a set of $k$ independent edges includes a spoke, then the remaining $k-1$ edges must be rim edges. The number of ways of choosing $k-1$ independent edges from a path with $p-2$ nodes is $\left(\begin{array}{c}p-k-1 \\ k-1\end{array}\right)$. Hence the number of elements in class $(i)$ is $(p-1)\left(\begin{array}{c}p-k-1 \\ k-1\end{array}\right)$.

If a set of $k$ independent edges does not include a spoke, then all the edges must be rim edges. The number of ways of choosing $k$ independent edges from a circuit with $p-1$ nodes, is $\frac{p-1}{k}\left(\begin{array}{c}p-k-2 \\ k-1\end{array}\right)$. (See Theorem 10 of [2]). Hence the number of sets of $k$ independent edges in $W_{p}$ is

$$
(p-1)\left(\begin{array}{c}
p-k-1 \\
k-1
\end{array}\right)+\frac{p-1}{k}\left(\begin{array}{c}
p-k-2 \\
k-1
\end{array}\right) .
$$

The result now follows.

Q.E.D.

The following theorem is obtained by substituting for $\mathrm{N}_{G}\left(\mathrm{n}_{1}, \mathrm{n}_{2}, \ldots, \mathrm{n}_{\mathrm{k}}\right)$ in the general expressions for $a_{k}$ and using Lemmas 1 and 2 .

THEOREM 8. The number of ways of covering the nodes of $w_{p}$ with $p-2(p \neq 4)$ nodedisjoint circuits is

$$
\frac{1}{2}(p-1)(3 p-8)
$$

With $\mathrm{p}-3(\mathrm{p} \neq 5)$ node-disjoint circuits it is

$$
(\mathrm{p}-1)\left[\left(\begin{array}{c}
\mathrm{p}-3 \\
2
\end{array}\right)+\frac{1}{3}\left(\begin{array}{c}
\mathrm{p}-5 \\
2
\end{array}\right)+1\right] \text {. }
$$

With $p-4(p \neq 6)$ node-disjoint circuits it is

$$
(p-1)\left[\left(\begin{array}{c}
p-5 \\
3
\end{array}\right)+\frac{1}{4}\left(\begin{array}{c}
p-6 \\
3
\end{array}\right)+p-4\right] \text {. }
$$


With $p-5(p \neq 7)$ node-disjoint circuits it is

$$
(p-1)\left[\left(\begin{array}{c}
p-5 \\
4
\end{array}\right)+\frac{1}{5}\left(\begin{array}{c}
p-7 \\
4
\end{array}\right)+p-5\right] .
$$

With $p-6(p \neq 8)$ node-disjoint circuits, it is

$$
(\mathrm{p}-1)\left[\left(\begin{array}{c}
\mathrm{p}-6 \\
5
\end{array}\right)+\frac{1}{6}\left(\begin{array}{c}
\mathrm{p}-8 \\
5
\end{array}\right)+\left(\begin{array}{c}
\mathrm{p}-6 \\
2
\end{array}\right)+1\right] \text {. }
$$

When $\mathrm{p}=4,5,6,7$ or 8 , we add 2 - $\mathrm{p}$ to the respective formula.

Using the general expressions for $b_{k}$ and Lemmas 1 and 2 we obtain simple expressions for the first 10 coefficients of the characteristic polynomial of the whee $1 \mathrm{~W}_{\mathrm{p}}$. The results are given in the following theorem.

THEOREM 9. Let

$$
\phi\left(W_{p} ; x\right)=\sum_{k=0}^{p} b_{k} x^{p-k}
$$

Then

and

$$
\begin{aligned}
& b_{0}=1, b_{1}=0, b_{2}=-(2 p-2), l_{3}=-(2 p-2) \quad(p \neq 4) \\
& b_{4}=\frac{1}{2}(p-1)(3 p-14) \quad(p \neq 5), \\
& b_{5}=2(p-1)(p-5) \quad(p \neq 6), \\
& b_{6}=(p-1)\left[2(p-6)-\left(\begin{array}{c}
p-4 \\
2
\end{array}\right)-\frac{1}{3}\left(\begin{array}{c}
p-5 \\
2
\end{array}\right)\right](p \neq 7), \\
& b_{7}=2(p-1)\left[\left(\begin{array}{c}
p-6 \\
1
\end{array}\right)-\left(\begin{array}{c}
p-5 \\
2
\end{array}\right)-3(p \neq 8),\right. \\
& b_{8}=(p-1)\left[\left(\begin{array}{c}
p-5 \\
3
\end{array}\right)+\frac{1}{4}\left(\begin{array}{c}
p-6 \\
3
\end{array}\right)-p+11 p-30\right](p \neq 9) . \\
& b_{9}=2(p-1)\left[\left(\begin{array}{c}
p-8 \\
1
\end{array}\right)-\left(\begin{array}{c}
p-7 \\
2
\end{array}\right)-\left(\begin{array}{c}
p-6 \\
3
\end{array}\right)-1\right](p \neq 10) .
\end{aligned}
$$

Theorem 9 can be used to obtain simplified expressions for the characteristics polynomials of wheels with up to 10 nodes. We note that a general formula for the characteristic polynomial of a wheel has been given in Cvetkovic [1].

\section{The Fan}

We define the fan $F_{p}$ to be the graph obtained by joining an isolated node to all the nodes of a path with $\mathrm{p}-1$ nodes.

LEMMA 3.

$$
\mathrm{N}_{\mathrm{F}}(\mathrm{n})=\mathrm{p}-\mathrm{n}+1 \text { for } \mathrm{n}>2 \text {. }
$$

$$
\mathrm{N}_{\mathrm{F}_{\mathrm{P}}}(2,2, \ldots, 2, \mathrm{n})=\sum_{\mathrm{r}=0}^{\mathrm{p}-\mathrm{n}} \sum_{1+j=k}\left(\begin{array}{c}
\mathrm{r}-1 \\
i
\end{array}\right)\left(\begin{array}{c}
p-n-r-j \\
j
\end{array}\right) \text {, }
$$

where $\mathrm{n}>2$ and $\mathrm{k}$ is the number of independent edges.

(iii) $\quad N_{F_{P}}\left(n_{1}, n_{2}, \ldots, n_{k}\right)=0$ if $n_{i}>2$ and $n_{j}>2$ for som $i$ and $j$, where

$$
1 \leqq i, j \leqq k \text {. }
$$

PROOF.

(i) and (iii) are straightforward. 
(ii) The n-gon will "use up" $n$ - 1 nodes on the rim of the fan.

The remaining graph will consist of two paths with $r$ and s edges, where $r+s=(p-1)-(n-1)$. Thus the $k$ independent edges must include $i$ from one path and $k$ - $i$ from the other, for $i=0,1,1, \ldots, k$. The result follows immediately.

LEMMA 4 .

Q.E.D.

$$
\mathrm{N}_{F_{P}}(2,2, \ldots, 2)=\left(\begin{array}{c}
p-k-2 \\
k
\end{array}\right)+\sum_{r+s=p-2}^{k} \sum_{i=0}^{k}\left(\begin{array}{c}
r-i \\
i
\end{array}\right)\left(\begin{array}{c}
s-k+i \\
k-i
\end{array}\right),
$$

where $\mathrm{k}$ is the number of independent edges.

PROOF. We can partition the independent edges into classes (i) those which contain only rim edges and (ii) those which do not. The number in Class (i) is $\left(\begin{array}{c}p-1-k \\ k\end{array}\right)$. The elements in Class (ii) can be counted by the technique used in establishing (ii) of Lemma 3 .

\section{Q.E.D.}

By using the general expressions for $a_{k}$ and Lemmas 3 and 4 we establish the following result.

THEOREM 10. The number of ways of covering the nodes of $F_{p}$ with $p-2$ nodedisjoint circuits is

$$
\left(\begin{array}{c}
\mathrm{p}-4 \\
2
\end{array}\right)+\sum_{\mathrm{r}+\mathrm{s}=\mathrm{p}-2}\left[\left(\begin{array}{c}
\mathrm{s}-2 \\
2
\end{array}\right)+(\mathrm{r}-1)(\mathrm{s}-3)+\left(\begin{array}{c}
\mathrm{r}-2 \\
2
\end{array}\right)\right]+\mathrm{p}-2
$$

With $\mathrm{p}-3$ node-disjoint circuits it is

$$
\begin{aligned}
\left(\begin{array}{c}
\mathrm{p}-5 \\
3
\end{array}\right)+ & \sum_{\mathrm{r}+\mathrm{s}=\mathrm{p}-2}\left[\left(\begin{array}{c}
\mathrm{s}-3 \\
3
\end{array}\right)+(\mathrm{r}-1)(\mathrm{s}-2)+\left(\begin{array}{c}
\mathrm{r}-2 \\
2
\end{array}\right)(\mathrm{s}-1)+\left(\begin{array}{c}
\mathrm{r}-3 \\
3
\end{array}\right)\right] \\
& +(\mathrm{p}-4)(\mathrm{p}-5)+(\mathrm{p}-3)
\end{aligned}
$$

With $p-4$ node-disjoint circuits it is

$$
\begin{aligned}
\left(\begin{array}{c}
p-6 \\
4
\end{array}\right)+ & \sum_{r+s=p-2} \sum_{i=0}^{4}\left(\begin{array}{c}
r-i \\
i
\end{array}\right)\left(\begin{array}{c}
s-4+i \\
4-i
\end{array}\right)+\sum_{r=0}^{p-3}\left[\left(\begin{array}{c}
p-5-r \\
2
\end{array}\right)\right. \\
& \left.+(r-1)(p-4-r)+\left(\begin{array}{c}
r-2 \\
2
\end{array}\right)\right]+(p-5)(p-6)+(p-4) .
\end{aligned}
$$

With $\mathrm{p}-5$ node-disjoint circuits it is

$$
\begin{aligned}
\left(\begin{array}{c}
p-7 \\
5
\end{array}\right)+ & \sum_{r+s=p-2} \sum_{i=0}^{5}\left(\begin{array}{c}
r-i \\
i
\end{array}\right)\left(\begin{array}{c}
s-5+i \\
5-1
\end{array}\right) \\
& +\sum_{r=0}^{p-3} \sum_{i+j=3}\left(\begin{array}{c}
r-i \\
i
\end{array}\right)\left(\begin{array}{c}
p-3-r-j \\
j
\end{array}\right)+\sum_{r=0}^{p-4}\left[\left(\begin{array}{c}
r-2 \\
2
\end{array}\right)\right. \\
& \left.+(r-1)(p-5-r)+\left(\begin{array}{c}
p-6-r \\
2
\end{array}\right)\right]+(p-6)(p-7)+(p-5) .
\end{aligned}
$$

With $\mathrm{p}-6$ node-disjoint circuits it is

$$
\begin{aligned}
\left(\begin{array}{c}
p-8 \\
6
\end{array}\right)+ & \sum_{r+s=p-2} \sum_{i=0}^{6}\left(\begin{array}{c}
r-i \\
i
\end{array}\right)\left(\begin{array}{c}
s-6+i \\
6-1
\end{array}\right)+\sum_{r=0}^{p-3} i+j=4 \\
+ & \sum_{r=0}^{p-4} \sum_{i+j=3}\left(\begin{array}{c}
r-i \\
i
\end{array}\right)\left(\begin{array}{c}
p-4-r-j \\
j
\end{array}\right) \\
& +(r-1)(p-6-r)+\left(\begin{array}{c}
p-7-r \\
2
\end{array}\right)+\sum_{r=0}^{p-5}\left[\left(\begin{array}{c}
r-2 \\
2
\end{array}\right)\right.
\end{aligned}
$$


The general expressions for $b_{k}$, together with Lemmas 3 and 4 yield the following result, which gives simple expressions for the first 10 coefficients of the characteristic polynomial of the fan $\mathrm{F}_{\mathrm{p}}$.

THEOREM 11. Let

$$
\phi\left(F_{p} ; x\right)=\sum_{k=0}^{p} b_{k} x^{p-k} .
$$

Then

$$
\begin{aligned}
& \mathrm{b}_{0}=1, \mathrm{~b}_{1}=0, \mathrm{~b}_{2}=-(2 \mathrm{p}-3), \mathrm{b}_{3}=-2(\mathrm{p}-2), \\
& \mathrm{b}_{4}=\left(\begin{array}{c}
\mathrm{p}-4 \\
2
\end{array}\right)+\sum_{\mathrm{r}+\mathrm{s}=\mathrm{p}-2}\left[\left(\begin{array}{c}
\mathrm{s}-2 \\
2
\end{array}\right)+(\mathrm{r}-1)(\mathrm{s}-3)+\left(\begin{array}{c}
\mathrm{r}-2 \\
2
\end{array}\right)\right]-2(\mathrm{p}-3), \\
& \mathrm{b}_{5}=2(\mathrm{p}-4)(\mathrm{p}-6) \text {, } \\
& \mathrm{b}_{6}=-\left[\left(\begin{array}{c}
\mathrm{p}-5 \\
3
\end{array}\right)+\sum_{\mathrm{r}+\mathrm{s}=\mathrm{p}-2}\left[\left(\begin{array}{c}
\mathrm{s}-3 \\
3
\end{array}\right)+(\mathrm{r}-1)(\mathrm{s}-2)+\left(\begin{array}{c}
\mathrm{r}-3 \\
3
\end{array}\right)\right]+2(\mathrm{p}-5)(\mathrm{p}-7)\right. \text {, } \\
& b_{7}=-2 \sum_{r=0}^{p-3}\left[\left(\begin{array}{c}
p-5-r \\
2
\end{array}\right)+(r-1)(p-4-r)+\left(\begin{array}{c}
r-2 \\
2
\end{array}\right)\right]+2(p-6)(p-8) \text {, } \\
& \mathrm{b}_{8}=\left(\begin{array}{c}
\mathrm{p}-6 \\
4
\end{array}\right)+\sum_{\mathrm{r}+\mathrm{s}=\mathrm{p}-2} \sum_{i=0}^{4}\left(\begin{array}{c}
\mathrm{r}-\mathrm{i} \\
\mathrm{i}
\end{array}\right)\left(\begin{array}{c}
\mathrm{s}-4+\mathrm{i} \\
4-\mathrm{i}
\end{array}\right)-2 \sum_{\mathrm{r}=0}^{\mathrm{p}-4}\left[\left(\begin{array}{c}
\mathrm{r}-2 \\
2
\end{array}\right)\right. \\
& \left.+(r-1)(p-5-r)+\left(\begin{array}{c}
p-6-r \\
2
\end{array}\right)\right]+2(p-7)(p-9)
\end{aligned}
$$

and

$$
\begin{aligned}
b_{9} & =-2 \sum_{r=0}^{p-3} \sum_{i+j=3}\left(\begin{array}{c}
r-i \\
i
\end{array}\right)\left(\begin{array}{c}
p-3-r-j \\
j
\end{array}\right)-2 \sum_{r=0}^{p-5}\left[\left(\begin{array}{c}
r-2 \\
2
\end{array}\right)+(r-1)(p-6-r)\right. \\
& \left.+\left(\begin{array}{c}
p-7-r \\
2
\end{array}\right)\right]+2(p-8)(p-10)
\end{aligned}
$$

\section{REFERENCES}

[1] CVEtkoví́, D.M. Graphs and their Spectra, Pub1. Elektrotehn Fak. Univ. Beograd, Ser. Mat. Fiz., Nos. 354-356 (1971) 1-50.

[2] FARRELL, E.J. On a Class of Polynomials Obtained from the Circuits in a Graph and its Application to Characteristic Polynomials of Graphs, Discrete Math. 25 (1979) 121-133.

[3] FARRELL, E.J. An Introduction to Matching Polynomials, J. Comb. Theory B 27 (1979) $75-86$.

[4] HARARY, F., KING, MOWSHOWITZ, A. and READ, R.C. Cospectral Graphs and Digraphs, Bu11. Lond. Math. Soc. 3 (1971) 321-328.

[5] SACHS, H. Beziehungen Zwischen den in einen Graphen enthaltenen Kreisen und seinen chaaracterisichen Polynom, Publ. Math. Debrecen 11 (1964), 119-134. 


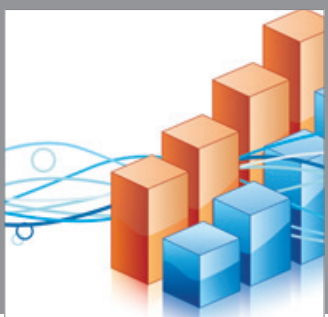

Advances in

Operations Research

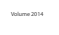

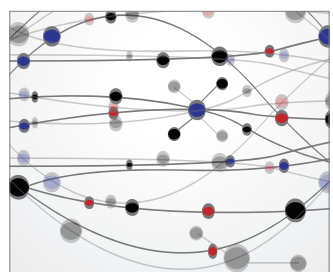

\section{The Scientific} World Journal
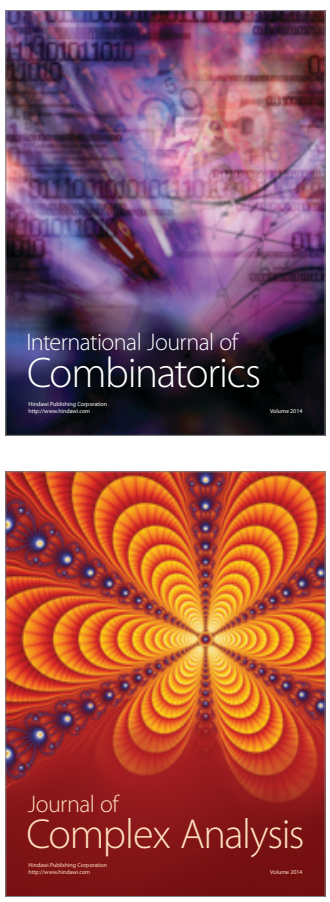

International Journal of

Mathematics and

Mathematical

Sciences
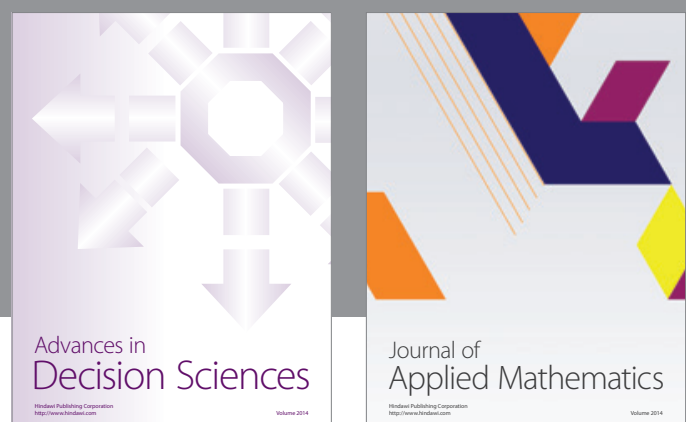

Journal of

Applied Mathematics
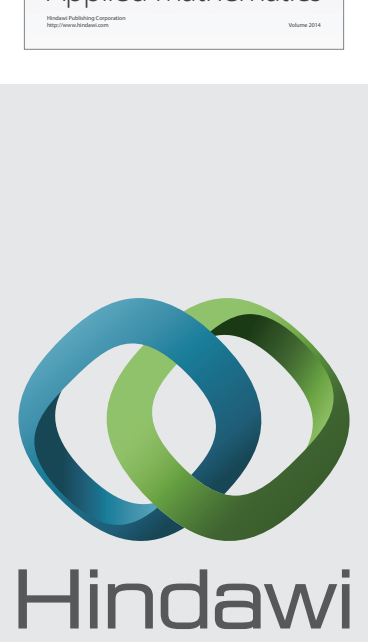

Submit your manuscripts at http://www.hindawi.com
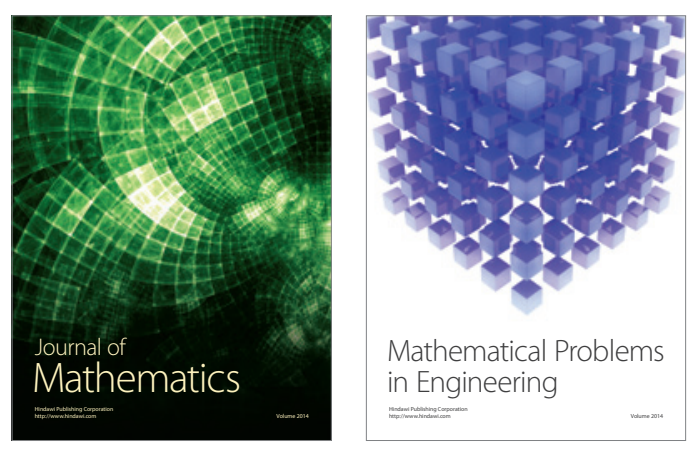

Mathematical Problems in Engineering
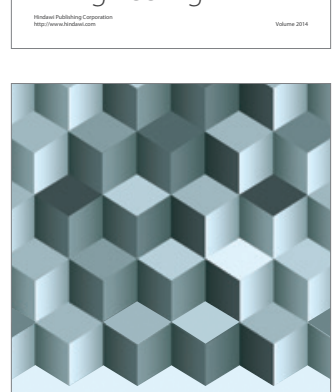

Journal of

Function Spaces
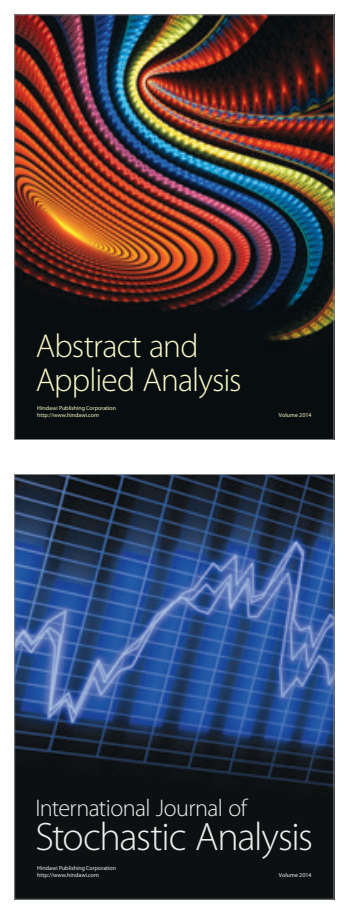

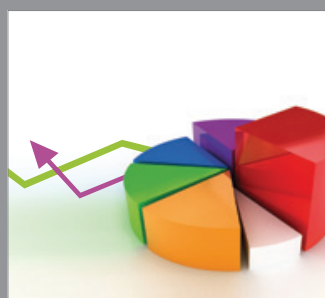

ournal of

Probability and Statistics

Promensencen
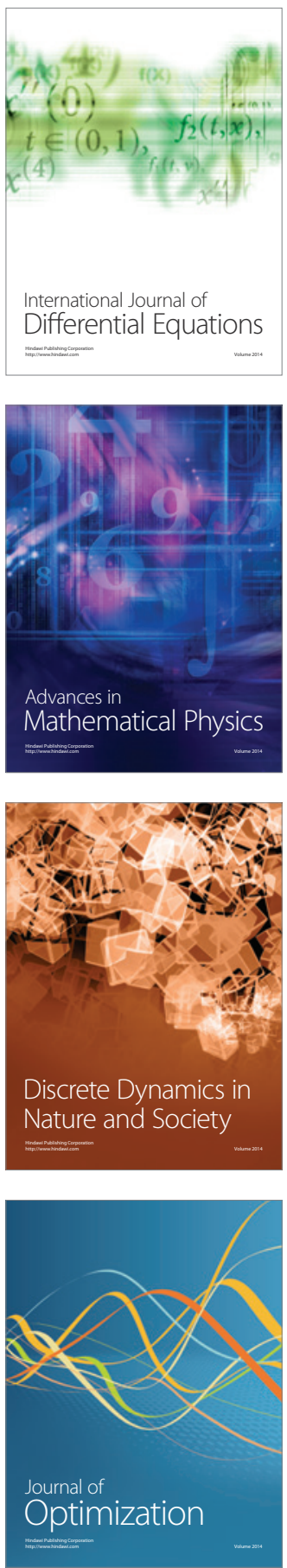